\title{
My patient is short of breath: is the problem in the lung tissue?
}

\author{
James Milne', Paul Atkinson ${ }^{1,2}$, Justin Bowra ${ }^{3}$, Osama Loubani, Bob Jarman ${ }^{5}$ \\ and Andrew Smith 6
}

${ }^{1}$ Dalhousie University - Medicine, Saint John, New Brunswick, E2L 4L5, Canada; ${ }^{2}$ Saint John Regional Hospital - Emergency Medicine, Saint John, New Brunswick, E2L 3L6, Canada; ${ }^{3}$ Sydney Adventist Hospital and Royal North Shore Hospital - Emergency Medicine, Wahroonga, New South Wales, 2076/2065, Australia; ${ }^{4}$ Dalhousie University - Emergency Medicine, Halifax, Nova Scotia, B3H 4R2, Canada; ${ }^{5}$ Queen Elizabeth Hospital - Emergency Medicine, Gateshead, NE9 6SX, UK; ${ }^{6}$ Memorial University - Emergency Medicine, St John's, Newfoundland, A1B 3U6, Canada

Corresponding author: Andrew Smith. Email: ajjsmith@me.com

\begin{abstract}
Undifferentiated dyspnoea is a common patient presentation in the intensive care unit, medical and surgical floors, and in the emergency department. Physical examination and chest radiography are notoriously insensitive for detection and differentiation of various lung pathologies while computed tomography consumes significant resources and exposes the patient to ionizing radiation. Point-of-care ultrasound (PoCUS) is a highly sensitive and specific diagnostic tool that, with appropriate operator experience, is capable of diagnosing and differentiating between the various causes of dyspnoea. PoCUS machines are readily available, images are rapidly generated and repeatable, and technical skills are easily taught during short training sessions. Furthermore, the development of PoCUS skills in one specific area enables and enhances the development of skills in other non-related areas. This article describes the benefits, technical aspects, and challenges associated with using PoCUS to examine the lung parenchyma in the acutely dyspnoeic patient.
\end{abstract}

Keywords: Ultrasound, lung, PoCUS, pulmonary oedema, pneumonia, COPD, interstitial syndrome, atelectasis

Ultrasound 2013; 0: 1-6. DOI: 10.1177/1742271X13486265

\section{Clinical questions}

Can point-of-care ultrasound (PoCUS) help me diagnose the cause of my patient's dyspnoea? Is it difficult to develop lung ultrasound skills or time consuming to generate the images? Can it really assist diagnosing pulmonary oedema or pneumonia more accurately than physical exam and chest radiography?

\section{Introduction}

Undifferentiated dyspnoea is a common patient presentation in many areas of acute care medicine. Traditionally, the history and physical examination, chest radiography, blood tests and the electrocardiogram have been integral to the diagnosis and management of patients with respiratory symptoms. PoCUS of the lung parenchyma provides an alternate way for physicians to rapidly diagnose respiratory conditions and guide management at the patient's bedside.

Some aetiologies of dyspnoea are relatively benign with comparatively low mortality rates; however, others have outcomes that are quite dire. For example, pulmonary contusions have mortality rates that range between $10 \%$ and $25 \%^{1}$ while community-acquired pneumonia requiring hospitalization has mortality rates of up to $15 \%{ }^{2,3}$ When comparing plain film and ultrasound to computed tomography (CT), only $27 \%$ of acute lung contusions will be detected (specificity $100 \%$ ) while PoCUS has a sensitivity of $94.6 \%$ and a specificity of $96.1 \%$, respectively. ${ }^{4}$ In patients with pneumonia, PoCUS has a sensitivity of $85.7 \%$ and a specificity of $97.7 \%$, while chest X-ray (CXR) was shown to be $69.2 \%$ sensitive and $85 \%$ specific. 5,6 Thus, PoCUS helps improve diagnostic certainty while guiding management, and has the potential to reduce mortality rates though the latter has not been substantiated by the literature.

The use of PoCUS in respiratory assessment has not been considered to be of great value until recently. ${ }^{5,7,8}$ Many sonographers correctly believed that an air filled lung interface would reflect the ultrasound waves, preventing any useful transmission of sound. However, with certain lung pathologies, there are regions devoid of air that often extend out to the pleura, so those ultrasound images of diseased lung are quite different than that of normal lung. These considerations provide the basis for the current use of lung ultrasound in modern medicine.

\section{How accurate is lung ultrasound?}

PoCUS is a modality that offers very high sensitivities and specificities for many respiratory conditions, often much 
Table 1 Sensitivity and specificity of chest radiography and PoCUS for various pulmonary conditions using CT as the gold standard

\begin{tabular}{|c|c|c|c|}
\hline & $\begin{array}{l}\text { Interstitial syndrome }{ }^{18} \\
\text { Sensitivity/Specificity (\%) }\end{array}$ & $\begin{array}{l}\text { Atelectasis }{ }^{19,20} \\
\text { Sensitivity/Specificity (\%) }\end{array}$ & $\begin{array}{l}\text { Alveolar consolidation }{ }^{6,18} \\
\text { Sensitivity/Specificity (\%) }\end{array}$ \\
\hline CXR & $46 / 80$ & $60 / 83$ & $38 / 89$ \\
\hline \multirow[t]{2}{*}{ PoCUS } & $94 / 93$ & $82 / 100$ & $86 / 78-98$ \\
\hline & $\begin{array}{l}\text { Pulmonary contusion }{ }^{4} \\
\text { Sensitivity/Specificity (\%) }\end{array}$ & $\begin{array}{l}\text { Tumour }^{21,22} \\
\text { Sensitivity/Specificity (\%) }\end{array}$ & $\begin{array}{l}\text { Abscess }^{23} \\
\text { Sensitivity/Specificity (\%) }\end{array}$ \\
\hline CXR & $27 / 100$ & $84 / 90$ & $19 / 94$ \\
\hline
\end{tabular}

CXR, chest X-ray; PoCUS, point-of-care ultrasound; CT, computed tomography

greater than traditional modalities such as the physical examination or chest radiography.

Chest radiography has a sensitivity of $69.2 \%$ when compared with high-resolution $\mathrm{CT}$ in the detection of community acquired pneumonia. ${ }^{6}$ When considering pulmonary oedema, physical examination findings such as a third heart sound, elevated jugular-venous pressure, rales or wheezes, new murmur and peripheral oedema tend to be fairly specific, but have very poor sensitivities ranging between $15 \%$ and $30 \% .{ }^{9}$ Similarly, findings on CXR also tend to be fairly specific, but only have sensitivities that range between $26 \%$ and $74 \% .{ }^{9}$ As clinicians, we depend on our history, physical examination, and diagnostic tests to form our clinical gestalt, yet over-reliance on findings with low sensitivities will likely lead to a significant proportion of misdiagnoses.

PoCUS demonstrates high sensitivities and specificities, using CT as the gold standard, for the diagnosis of many pulmonary conditions including pneumothorax (sensitivity $86-98 \%$, specificity $97-99 \%{ }^{10,11}$ ), pleural effusion (sensitivity $92 \%$, specificity $93 \%{ }^{12}$ ), pulmonary oedema (sensitivity $97 \%$, specificity $94 \%{ }^{5}$ ) and pneumonia (sensitivity $89 \%$, specificity $94 \%{ }^{13}$ ). These, along with other pathologies, are shown in Table 1. Lung ultrasound has also been used to detect pulmonary embolism with some success in several research studies, but is an advanced, controversial topic. ${ }^{14,15}$ Details regarding use of ultrasound to detect pneumothorax and pleural effusion are the focus of other papers in this series. ${ }^{16,17}$

As with other PoCUS indications, the techniques described in this article are not meant to be a substitute for the physical examination, diagnostic radiology or a physician's clinical impression. Rather, it is another tool capable of providing accurate, supplemental information via a readily available, non-invasive tool. In order to achieve sensitivities and specificities similar to those presented in Table 1 and to ensure appropriate clinical use, a sound understanding of proper technique, the physics of sonography, and a methodical approach are important.

\section{Point-of-care ultrasound}

The technique

Scanning the thorax should be performed in a methodical fashion to rule-in or rule-out pulmonary pathology, depending on the clinical question being asked. Volpicelli ${ }^{5}$

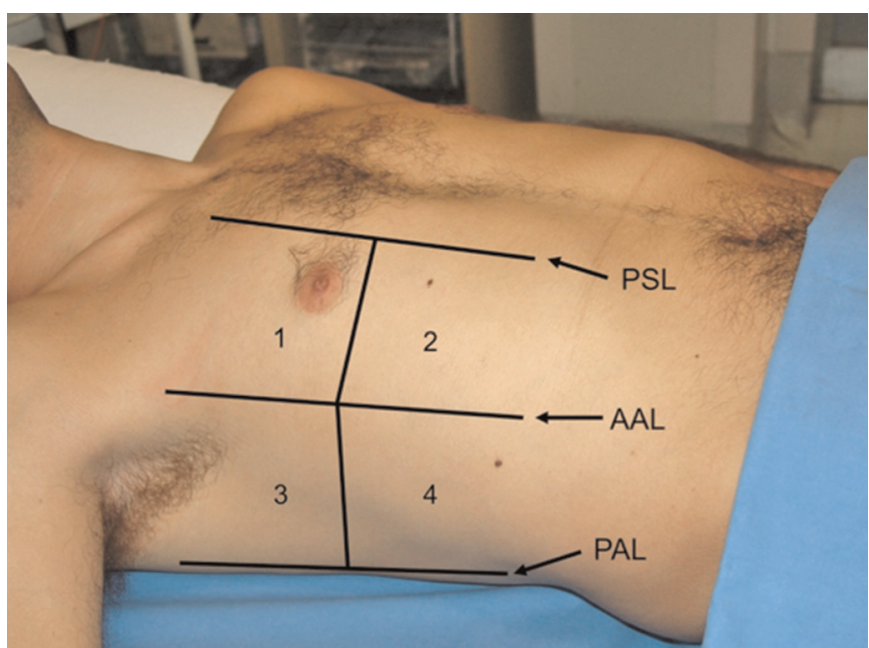

Figure 1 Each hemithorax is divided into four quadrants to ensure a methodical assessment of the lung parenchyma. This helps differentiate between a diffuse or localized process. PSL, parasternal line; AAL, anterior axillary line; PAL, posterior axillary line (modified from Volpicelli et al. with permission) ${ }^{5}$

described an approach to lung ultrasound by dividing each hemithorax into quadrants and scanning each as shown in Figure 1.

The probe is typically oriented in the longitudinal plane with the probe marker pointed cephalad. The ribs act as sonographic landmarks enabling quick and accurate identification of the pleura as shown in Figure 2. More advanced sonographers may experiment with oblique probe orientations in the attempt to visualize more pleura.

The patient may be scanned in either the supine or sitting position. At least one scan should be taken from each quadrant for a total of eight scans. Additional scans of the two posterior regions can be included when the patient is sitting or leaning forward (as is often the case in severe respiratory distress). The sonographer should attempt to scan the most dependent portion of the lung, as shown in Figure 3, as it is here that pathology becomes most evident. ${ }^{7}$

\section{Transducer selection and machine settings}

Any ultrasound probe can be used to image the lung tissue, though there are advantages and disadvantages to each. The curvilinear probe $(1-5 \mathrm{MHz})$, the most widely available, is well suited to this task mainly due to the low frequency, which provides good depth and tissue penetration. The main drawback is the relatively large 


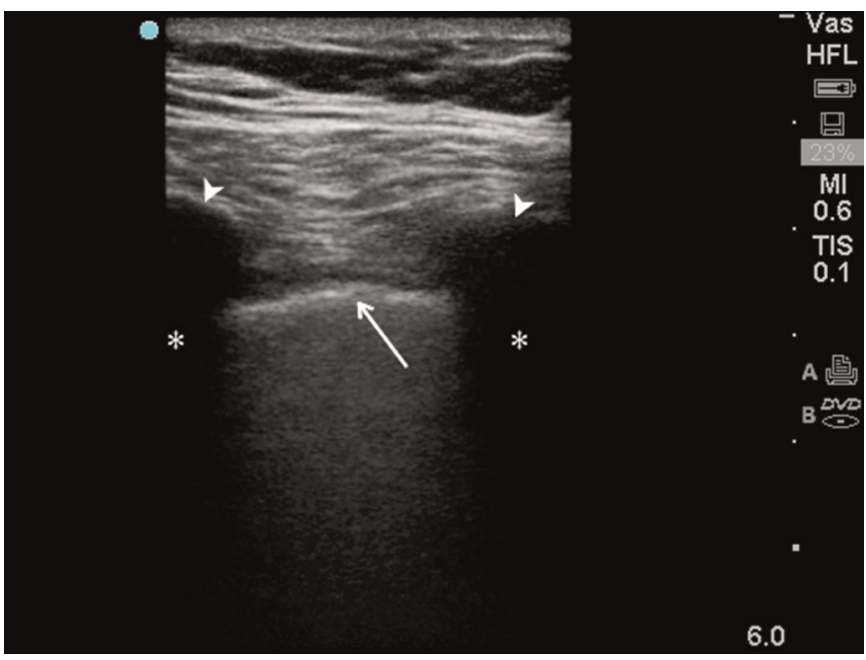

Figure 2 Typical sonographic image of the lung using a linear, high frequency probe. The ribs (arrowheads), acoustic shadowing (asterix) and pleural line (arrows) are all clearly visible

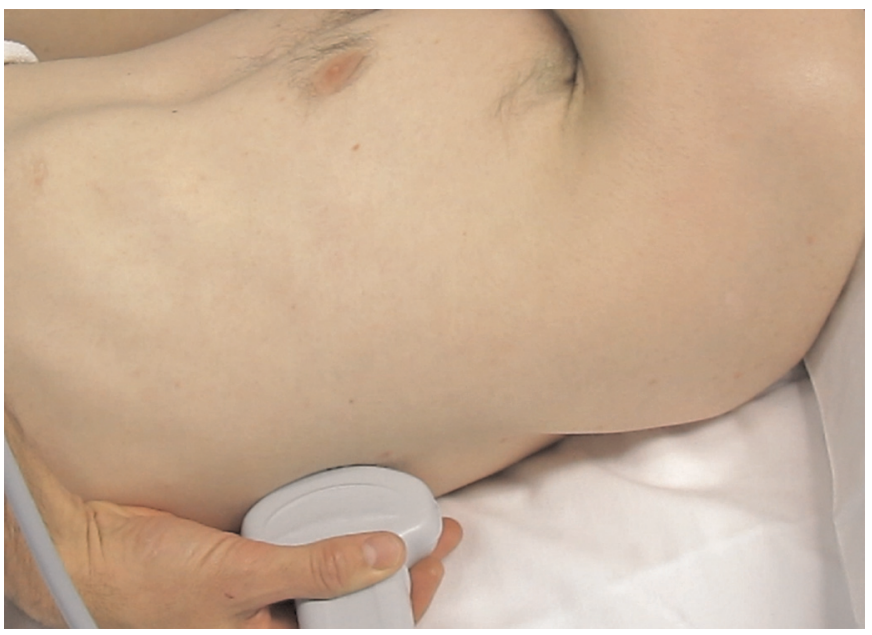

Figure 3 Scanning the most dependent portion of the lungs provides the highest yield in detecting pathology. Here the probe is oriented in the longitudinal plane with the probe marker pointed cephalad footprint, which is difficult to fit neatly into the rib spaces. The micro-convex probe $(2-8 \mathrm{MHz}$; manufacturer dependent) is the most ideal for imaging the lung as it has a small footprint, a wide field of view and a low- to mid-range frequency. The major drawback of this probe is that it is not available in most settings, and as such many sonographers are unfamiliar with it. The linear transducer $(8-12 \mathrm{MHz})$ is best for imaging the pleura and performing guided procedures due to its high frequency; however, it is unable to provide information about structures at a greater depth. The phased-array transducer $(1-5 \mathrm{MHz})$ has a small footprint that allows it to fit between the ribs, but its poor spatial resolution (particularly in the near field) means that it is not as suitable for lung scanning.

Traditionally, novice sonographers spend significant time learning about various ultrasound artefacts and how to minimize their impact on generated images. Modern ultrasound machines use sophisticated software to 'smooth' the ultrasound images and minimize artefacts. However, this actually decreases the overall ability to detect pulmonary pathology as the identification of various artefacts is important. Therefore, many of these advanced functions (specifically tissue harmonic imaging [THI] and compound imaging, e.g. multi-beam/crossbeam) should be turned off for the purposes of lung ultrasound, particularly if scanning for B-line artefacts (laser-like lines that extend from the pleura to the bottom of the screen). By way of example, Figure 4a demonstrates how THI can impair the operator's ability to clearly identify the B-lines all the way to the bottom of the screen. Figure $4 \mathrm{~b}$ shows the same patient, same site, approximately 15 seconds later with THI off and B-lines clearly visible.

\section{Normal sonographic lung tissue}

When scanning normal lung, little information about the actual parenchyma is seen, due to the almost complete reflection of sound at the aerated lung-pleura interface. This is due to the presence of air. The bright line of the pleura - shown in Figure 5 - appears to connect the two adjacent ribs with little beyond other than a sea of grey fog.
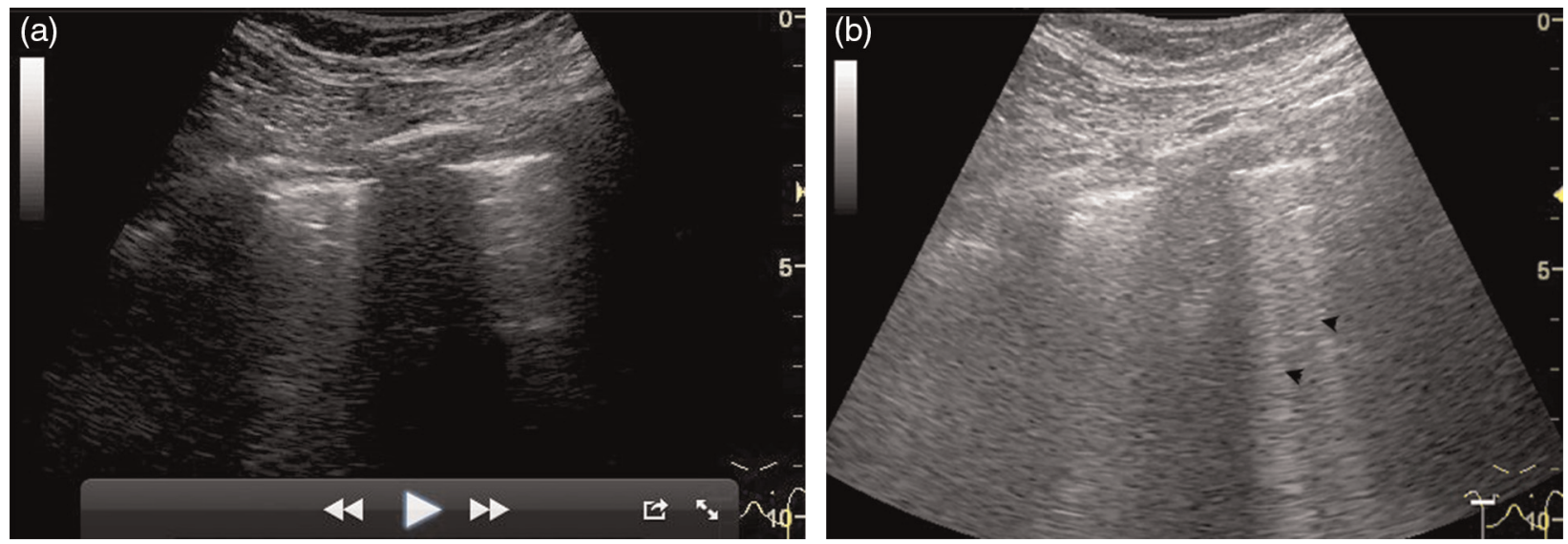

Figure 4 (a) B-lines with THI on. (b) B-lines with THI off. The arrowheads point to the B-lines - laser-like projections that extend to the bottom of the screen and move with lung sliding - which are seen much more clearly when compared with (a) 


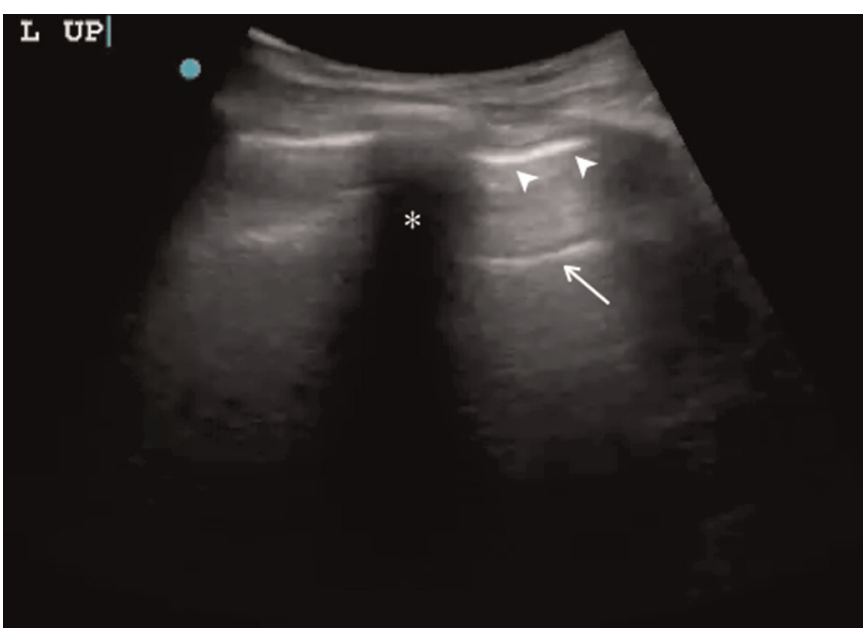

Figure 5 A-lines are a reverberation artefact representing reflections of the pleural line deeper in the lung tissue. These are found in two-thirds of normal subjects. The pleura (arrowheads), rib shadow (asterix) and A-line (arrow) as seen using a curvilinear probe

Often 'A-lines' are seen. These are reverberation artefacts, representing parallel repeats of the pleural line deeper into the field. These are seen in two-thirds of normal subjects. It is of interest to note that an ultrasound image of the normal lung is no different than that of the emphysematous lung as both are well aerated.

\section{Specific pathologies}

Each specific lung pathology has findings that differentiate it from other aetiologies of dyspnoea. This section examines some common parenchymal pathologies associated with shortness of breath, describes the ultrasound findings and provides some sample images.

\section{Interstitial syndrome}

Acute pulmonary oedema frequently presents as severe respiratory distress and can be difficult to differentiate from acute exacerbations of chronic obstructive pulmonary disease (COPD). Lichtenstein and Meziere ${ }^{24}$ first discovered that lung ultrasound could be used to differentiate between these two conditions with a very high sensitivity and specificity through the detection of B-lines. These B-line artefacts, previously referred to as comet tails, are thought to represent the reverberation of sound waves at the pleural surface due to increased extravascular fluid and air bubbles. ${ }^{25}$ The term interstitial syndrome is an umbrella term used to describe the findings of diffuse B-lines involving both lungs. Cardiogenic and non-cardiogenic pulmonary oedema, interstitial pneumonia and pulmonary fibrosis fall into this category.

Cardiogenic pulmonary oedema is a hydrostatic oedema, characterized by many diffuse, symmetric B-lines, with increased prevalence in dependent areas of the lung, and the often present pleural effusions. Lung sliding, or the apparent movement of the pleural line, is preserved or even aided by the presence of pleural transudate. ${ }^{19,26}$

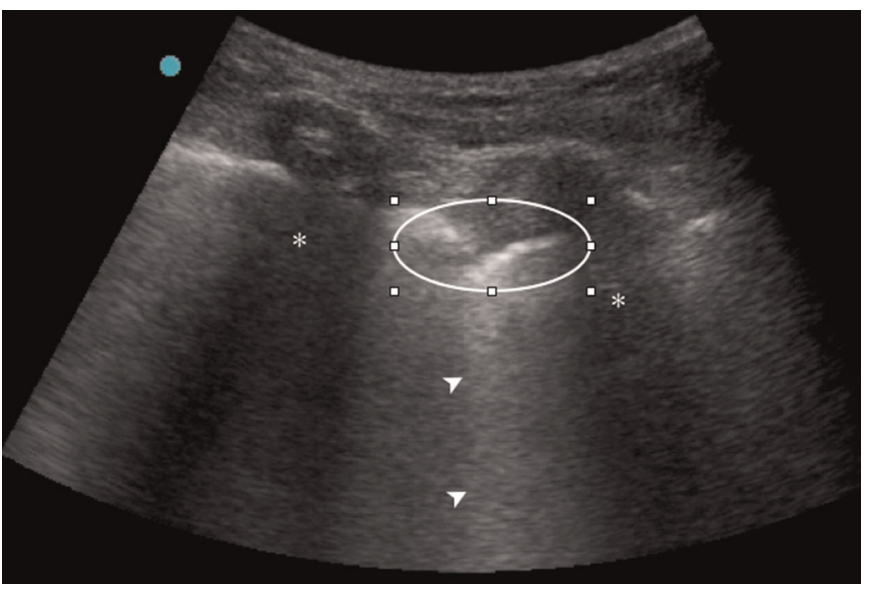

Figure 6 A microconsolidation (circle) creating an irregularity of the pleural line along with an associated B-line (arrowheads). Rib shadows are once again depicted (asterix)

By contrast, pulmonary oedema due to acute respiratory distress syndrome (ARDS) and interstitial pneumonia is an inflammatory oedema. As such, it is typically patchy with areas of normal, spared lung (ARDS) or localized (pneumonia). Lung sliding is often diminished or absent (retarded by the presence of a 'sticky' proteinaceous pleural exudate). Close examination of the pleura may reveal irregularities (microconsolidations and/or infarcts) and there may also be larger areas of subpleural consolidation as shown in Figure $6 .{ }^{19}$

Pulmonary fibrosis will also cause prominent diffuse B-lines. This tends to be associated with a thickened, irregular pleural line and diminished sliding. ${ }^{26}$

The sonographer must remember that the above conditions may coexist and can resemble each other on ultrasound. In such cases, clinical assessment will often provide clues as to which pathology is paramount.

PoCUS experts have recently developed an evidencebased consensus statement regarding the interstitial syndrome and other applications of lung ultrasound. They defined a positive quadrant as representing three or more B-lines in the vertical space between two ribs and a positive examination as being two or more positive quadrants bilaterally. ${ }^{27}$ This pattern is strongly suggestive of diffuse interstitial syndrome, whereas focal, positive quadrant scans can be suggestive of normal lung tissue or other localized pathology. ${ }^{28}$

\section{Atelectasis}

Lung tissue devoid of air provides an ideal path for the transmission of ultrasound waves. In regions of complete atelectasis, the lung parenchyma will appear similar to other solid organs such as the liver or spleen and is often referred to as 'hepatization'. In partial atelectasis there exist small pockets of air trapped within individual or multiple alveoli (a static air bronchogram) that are displayed as hyperechoic foci within the hepatisized lung as shown in Figure 7. Other findings include diminished or absent lung sliding, along with the presence of a lung 'pulse', the 


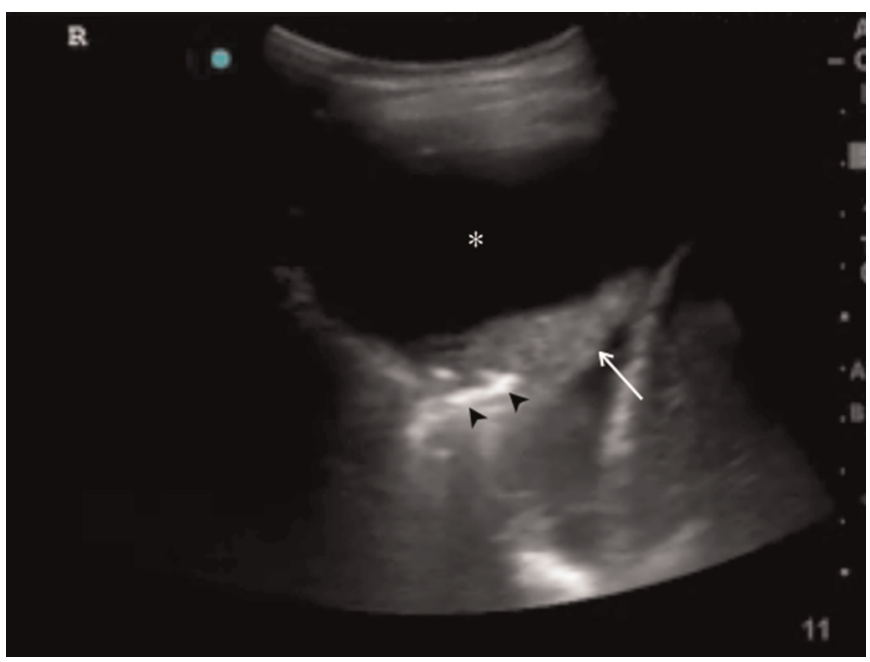

Figure 7 An atelectatic lung tip suspended in a pleural effusion (asterix). The hepatisized lung (arrow) is with static air bronchograms (arrowheads)

mechanical transmission of cardiac contractility through the lung parenchyma. $^{29}$

\section{Alveolar consolidation}

Differentiating between alveolar consolidation and atelectasis can be a little more challenging, as these conditions frequently have similar findings. One marked difference is the presence of dynamic air bronchograms. Differentiating between a static and dynamic air bronchogram, as seen in consolidations and virtually never in atelectasis, is best seen on a video displayed on the Emergency Ultrasound channel, YouTube. ${ }^{30}$ The dynamic air bronchogram has a specificity of $94 \%$ for consolidation with a positive predictive value of $97 \% .^{31}$ Note should be made that occasionally, heavy consolidation can be devoid of any bronchograms and potentially mistaken for a fluid collection or mass.

\section{Other pathologies}

Other causes of dyspnoea include pulmonary contusion, infarction, tumour and abscess. If the pathology apposes the pleural surface of the lung, these conditions can be detected using ultrasound (though their appearance can be quite variable). Unlike atelectasis and consolidation, air bronchograms will not be demonstrable. An abscess may be visible as a fluid filled area, and at times a tumour will as well (because of areas of necrosis). Although PoCUS can detect abscesses and tumours, it under-performs the chest radiograph and CT, as shown in Table 1, as these pathologies frequently occur in deep lung tissue.

\section{Challenges}

There are several aspects of lung ultrasound that should be considered, as they impact skill development and adoption into clinical practice. First of all, the practice of lung ultrasound places increased time demands on the practitioner as opposed to the simple ordering of a chest radiograph or CT scan. With experience, the complete lung ultrasound examination can be performed within three minutes. Secondly, the occasional B-lines are present in the normal lung - particularly the dependent zones. Finally, any PoCUS examination is user-dependent and is only as good as the operator's knowledge and skill. It is important to ensure that the results of clinical ultrasounds are always taken in the context of the history and physical examination and other investigations.

\section{DECLARATIONS}

Competing interests: None.

Funding: None.

Ethical approval: Not applicable.

Guarantor: JM.

Contributorship: JM performed the initial literature review, composed the first draft, made revisions recommended by other authors and completed the initial submission process. He also composed and submitted the revised final draft. PA revised the first and final drafts, acted as a general supervisor throughout the composition process. JB revised the initial paper and provided the majority of images used within. OL revised the initial draft. BJ revised the initial and final draft. AS was major contributor to first draft, revisions to initial and final versions as well as formatted citations. He completed suggested recommendations and was acting senior author. All authors have reviewed this final version of the article and have accepted it.

\section{REFERENCES}

1 Hoff SJ, Shotts SD, Eddy VA, Morris JA Jr. Outcome of isolated pulmonary contusion in blunt trauma patients. Am Surg 1994;60:138-42

2 Carriere KC, Jin Y, Marrie TJ, Predy G, Johnson DH. Outcomes and costs among seniors requiring hospitalization for community-acquired pneumonia in Alberta. J Am Geriatr Soc 2004;52:31-8

3 Niederman MS, McCombs JS, Unger AN, Kumar A, Popovian R. The cost of treating community-acquired pneumonia. Clin Ther 1998;20:820-37

4 Soldati G, Testa A, Silva F, Carbone L, Grazia P, Silvera N. Chest ultrasonography in lung contusion. Chest 2006;130:533-8

5 Volpicelli G, Mussa A, Garofalo G, et al. Bedside lung ultrasound in the assessment of alveolar-interstitial syndrome. Am J Emerg Med 2006;24:689-96

6 Cortellaro F, Colombo S, Coen D, Duca PG. Lung ultrasound is an accurate diagnostic tool for the diagnosis of pneumonia in the emergency department. Emerg Med J 2012;29:19-23

7 Zanobetti M, Poggioni C, Pini R. Can chest ultrasonography replace standard chest radiography for evaluation of acute dyspnea in the ED? Chest 2011;139:1140-7

8 Lichtenstein D. General Ultrasound in the Critically Ill. Berlin, Haidelberg, New York: Springer-Verlag, 2005

9 Wang CS, FitzGerald JM, Schulzer M, Mak E, Ayas NT. Does this dyspneic patient in the emergency department have congestive heart failure? JAMA 2005;294:1944-56

10 Zhang M, Liu ZH, Yang JX, et al. Rapid detection of pneumothorax by ultrasonography in patients with multiple trauma. Crit Care 2006;10:R112

11 Blaivas M, Lyon M, Duggal S. A prospective comparison of supine chest radiography and bedside ultrasound for the diagnosis of traumatic pneumothorax. Acad Emerg Med 2005;12:844-9

12 Lichtenstein D, Goldstein I, Mourgeon E, Cluzel P, Grenier P, Rouby JJ. Comparative diagnostic performances of auscultation, chest 
radiography, and lung ultrasonography in acute respiratory distress syndrome. Anesthesiology 2004;100:9-15

13 Lichtenstein DA, Lascols N, Meziere G, Gepner A. Ultrasound diagnosis of alveolar consolidation in the critically ill. Intensive Care Med 2004;30:276-81

14 Mathis G, Blank W, Reissig A, et al. Thoracic ultrasound for diagnosing pulmonary embolism: a prospective multicenter study of 352 patients. Chest 2005;128:1531-8

15 Volpicelli G, Cardinale L, Berchialla P, Mussa A, Bar F, Frascisco MF. A comparison of different diagnostic tests in the bedside evaluation of pleuritic pain in the ED. Am J Emerg Med 2012;30:317-24

16 Loubani O, Atkinson P, Smith A. My patient is short of breath - is there pleural fluid and can I drain it safely? Ultrasound 2013;21

17 Lewis D. My patient is short of breath: have they got a pneumothorax? Ultrasound 2011;19:230-5

18 Xirouchaki N, Magkanas E, Vaporidi K, et al. Lung ultrasound in critically ill patients: comparison with bedside radiography. Intensive Care Med 2011;37:1488-93

19 Orji FT, Akpeh JO. Tracheobronchial foreign body aspiration in children: how reliable are clinical and radiological signs in the diagnosis? Clin Otolaryngol 2010;35:479-85

20 Yang J, Zhang M, Liu Z, Ba L, Gan J, Xu S. Detection of lung atelectasis/consolidation by ultrasound in multiple trauma patients with mechanical ventilation. Crit Ultrasound J 2009;1:13-6

21 Toyoda Y, Nkayama T, Kusinoki Y, Iso H, Suzuki T. Sensitivity and specificity of lung cancer screening using chest low-dose computed tomography. Br J Cancer 2008;98:1602-07
22 Koh DM, Burke S, Davies N, Padley SP. Transthoracic US of the chest: clinical uses and applications. Radiographics 2002;22:e1

23 Voggenreiter G, Aufmkolk M, Majetschak M, et al. Efficiency of chest computed tomography in critically ill patients with multiple traumas. Crit Care Med 2000;28:1033-39

24 Lichtenstein D, Meziere G. A lung ultrasound sign allowing bedside distinction between pulmonary edema and COPD: the comet-tail artifact. Intensive Care Med 1998;24:1331-4

25 Soldati G, Copetti G, Sher S. Sonographic interstitial syndrome: the sound of lung water. J Ultrasound Med 28:163-74

26 Reissig A, Kroegel C. Transthoracic sonography of diffuse parenchymal lung disease: the role of comet tail artifacts. J Ultrasound Med 2003;22:173-80

27 Volpicelli G, Elbarbary M, Blaivas M, et al. International evidence-based recommendations for point-of-care lung ultrasound. Intensive Care Med 2012;38:577-91

28 Volpicelli G, Caramello V, Cardinale L, Mussa A, Bar F, Frascisco MF. Detection of sonographic B-lines in patients with normal lung or radiographic alveolar consolidation. Med Sci Monit 2008;14:CR122-8

29 Lichtenstein DA, Lascols N, Prin S, Meziere G. The 'lung pulse': an early ultrasound sign of complete atelectasis. Intensive Care Med 2003;29:2187-92

30 Dynamic Air Bronchogram. See http://www.youtube.com/watch?v =9Ra1QuqBY-8 (last checked December 2012)

31 Lichtenstein D, Meziere G, Seitz J. The dynamic air bronchogram. A lung ultrasound sign of alveolar consolidation ruling out atelectasis. Chest 2009;135:1421-5 